\title{
Laser Assisted Extraction of Pacemaker and Implantable Defibrillator Leads
}

\author{
Roberto Costa, Martino Martinelli Filho, Elizabeth Sartori Crevelari, Noedir Antonio Groppo Stolf, \\ Sérgio Almeida de Oliveira
}

São Paulo, SP - Brazil

\begin{abstract}
Objective - To analyze the results of laser-assisted extraction of permanent pacemaker and defibrillator leads.

Methods - We operated upon 36 patients, whose mean age was 54.2 years, and extracted 56 leads. The reasons for extracting the leads were as follows: infection in $19 \mathrm{pa}$ tients, elective replacement in 13, and other causes in 4 patients. The mean time of catheter placement was $7.5 \pm 5.5$ years. Forty-seven leads were from pacemakers, and 9 were from defibrillators. Thirty-eight leads were in use, 14 had been abandoned in the pacemaker pocket, and 4 had been abandoned inside the venous system.
\end{abstract}

Results - We successfully extracted 54 catheters, obtaining a $96.4 \%$ rate of success and an $82.1 \%$ rate for complete extraction. The 2 unsuccessful cases were due to the presence of calcium in the trajectory of the lead. The mean duration of laser light application was $123.0 \pm 104.5 \mathrm{~s}$, using 5,215.2 $\pm 4,924$.0 pulses, in a total of $24.4 \pm 24.2 \mathrm{cy}$ cles of application. Thirty-four leads were extracted from the myocardium with countertraction after complete progression of the laser sheath, 12 leads came loose during the progression of the laser sheath, and the remaining 10 were extracted with other maneuvers. One patient experienced cardiac tamponade after extraction of the defibrillator lead, requiring open emergency surgery.

Conclusion - The use of the excimer laser allowed extraction of the leads with a $96 \%$ rate of success; it was not effective in 2 patients who had calcification on the lead. One patient (2.8\%) had a complication that required cardiac surgery on an emergency basis.

Key words: artificial cardiac pacemaker, lead extraction, laser light

Instituto do Coração do Hospital das Clínicas - FMUSP

Mailing address: Roberto Costa - InCor - Av. Dr. Enéas C. Aguiar, 44 -05403-000

- São Paulo, SP, Brazil - E-mail: rcosta@ incor.usp.br

English version by Stela Maris C. e Gandour
The extraction of venous leads of permanent pacemakers and defibrillators poses a great challenge to artificial cardiac stimulation, because of the high indices of failure and complications observed with the different techniques used.

The clinical conditions requiring extraction of these catheters are luckily not frequent and include infection in the stimulation system, venous thrombosis, repetitive pulmonary embolisms, or other situations associated with the presence of a catheter and that constitute a risk to the patient's life ${ }^{1,2}$.

The routine practice of abandoning leads with no function has also become a problem with the use of multiple electrodes of the dual-chamber and multisite pacemakers. In the same way, the electrodes of implantable defibrillators, because of their large caliber and rough surface, also cause a severe problem when they require replacement or extraction. This management increases the risk of pulmonary thromboembolism, syndrome of the superior vena cava, and, frequently, hinders the access route for placement of new leads.

Three major approaches for extracting venous electrodes have been used as follows: 1) direct external traction of the leads, which has a low success rate and a high risk of laceration of the cardiac and venous structures; 2) cardiotomy, usually performed with the aid of cardiopulmonary bypass; and 3 ) internal countertraction, which is performed with sheaths of Teflon or polypropylene with a good success rate ${ }^{1}$.

Internal countertraction is a transvenous technique, and, consequently, less invasive than cardiotomy, therefore, being the preferred approach in most services. It requires, however, the introduction of a sheath that releases the lead to be extracted, and advances in the vein, encircling the lead until reaching the cardiac chamber, where the electrode is implanted, to act as support for the myocardium during lead extraction. The introduction of this sheath may be performed using either mechanical dilators that break the fibrous tissue throughout the trajectory of the electrode or the excimer laser that cuts this fibrosis. 
Recent studies published in the literature have shown the superiority of the technique that uses the laser light, which has a higher rate of success in removing the fibrosis and a lower rate of complications.

The objective of this study is to report the results obtained with the use of the excimer laser for extracting permanent transvenous leads from 36 patients operated upon at the of the University of São Paulo. Heart Institute (Medical School)

\section{Methods}

During the period from September 1998 to June 2000, we operated upon 36 patients with ages ranging from 1 to 91 years (mean $=54.2 \pm 21.9$ years). Twenty-two patients were males and 14 were females. The causes of the disorder in the cardiac conduction system were as follows: Chagas' disease in 9 patients, degenerative diseases in 6 patients, ischemic disease in 5 , and other causes in 16 patients. At the moment of extraction, 23 patients were bradycardic because of atrioventricular block in 17 patients and sinus dysfunction in 6 patients.

The reason for extracting the leads was infection in the stimulation system in 19 patients, elective replacement of the electrode in 13, and other causes in 4 (tab. I).

The catheters extracted had been implanted from 8 months to 21.8 years (mean $=90 \pm 66$ months) previously, 47 being pacemaker leads and 9 being defibrillator leads. The leads had been in place longer in the group of patients with pacemakers (mean $=103.2 \pm 63.6$ months). Forty-two leads had been implanted in the right ventricle and 14 in the right atrium. In the patients with defibrillators, the electrodes had been in place for a shorterperiod (mean $=24.0 \pm 10.8$ months) (tab. II).

\begin{tabular}{|lc|}
\hline \multicolumn{2}{|c|}{ Table I - Clinical characteristics of the patients } \\
\hline Number of patients & 36 \\
Age (years) & $54.2 \pm 21.9$ \\
Female/male sex & $14 / 22$ \\
$\quad$ - Mandatory indication & 3 \\
Endocarditis/septicemia & \\
$\quad$ - Necessary indication & 6 \\
Abscess in the pacemaker pocket & 9 \\
Skin erosion & 1 \\
Unclear fever & \\
$\quad$ Optional indications & 2 \\
Discontinuity & 13 \\
Elective replacement & 2 \\
Other causes & \\
\hline
\end{tabular}

\begin{tabular}{|lc|}
\hline \multicolumn{2}{|c|}{ Table II - Characteristics of the extracted leads } \\
\hline Electrodes per patient & 1.55 \\
Atrial/ventricular & $14 / 42$ \\
ICD/PM & $9 / 47$ \\
Length of stay (months) & $90 \pm 66$ \\
\hline ICD- implantable cardioverter defibrillator; PM- pacemaker. \\
\hline
\end{tabular}

The access route through which the leads had been implanted was the cephalic vein for 10 leads, the subclavian vein for 40 , the jugular vein for 3 , and the femoral vein for 3 leads.

At the time when extraction was indicated, 38 leads were being used, 14 had been abandoned in the pacemaker pocket, and 4 had been abandoned inside the venous system.

General anesthesia was used in 34 patients, and local anesthesia associated with sedation was used in 2 patients.

The CVX-300 excimer laser emits a beam of light of 308 $\mathrm{nm}$, which is not visible to the human eye. It is a type of laser light that cuts using coldness: the temperature of the light emitted is approximately $50^{\circ} \mathrm{C}$. At the tissue depth of approximately $0.06 \mathrm{~mm}, 64 \%$ of the energy is absorbed, and at the tissue depth of $0.18 \mathrm{~mm}, 95 \%$ of this energy is dissipated. Light is absorbed by lipids and proteins, but not by water, which is the major dissipation medium of other laser light modalities; this allows photoablation of the fibrous bands that surround the lead without damaging it, and with no harm to the venous and cardiac structures.

The beams of light are emitted by the distal extremity of flexible sheaths that are passed above the electrode to be extracted, completely encircling it after its release from the fibrous tissue, providing circumferential support to the tip of the electrode in the myocardium for the application of internal countertraction. The body of this sheath contains 82 optical fibers that conduct the laser light as far as their distal portions, each one with a diameter of $100 \mathrm{~mm}$. Progression of the laser sheath upon the lead is obtained as the light beam cuts the fibrous tissue, and it is followed by continuous fluoroscopy. Total release of the lead is obtained when the sheath reaches the endocardium, in the cardiac chamber where the electrode is implanted.

Three different calibers of sheath exist: 12,14, and 16F, corresponding respectively to $2.8 \mathrm{~mm}, 3.4 \mathrm{~mm}$, and $4.2 \mathrm{~mm}$ of internal diameter, and $4.1 \mathrm{~mm}, 4.8 \mathrm{~mm}$, and $5.6 \mathrm{~mm}$ of external diameter. The proper choice of caliber of the sheath allows the extraction of any model of lead used in artificial venous cardiac stimulation.

Patients are preferably operated upon while they are under general anesthesia with orotracheal intubation. In the horizontal dorsal decubitus position, under continuous monitoring of blood pressure and electrocardiography, the patients receive adhesive plates for cardiac stimulation and percutaneous defibrillation. Antisepsis of the skin is performed with povidone iodine, and sterile fields are placed to allow an easy access for sternal or lateral median thoracotomy. All material for thoracotomy should be easily accessible.

After opening the pacemaker pocket, and with the electrode already released from the generator of pulses, the lead is carefully dissected from the scare tissue that envelopes it, and the suture sleeve is extracted. A smooth manual traction is then applied to check whether the extraction is feasible without using the set of extraction sheaths, and with no damage to the lead. If the manual extraction is not possible, the extraction procedure proceeds. 
The standard procedure for extracting the lead starts with extraction of the connector of the lead with a sterile wire cutter, and a long proximal portion of the lead is left to allow handling of the locking stylet and sheaths. As the distal part of the conductor may be deformed by the use of the wire cutter, a sharp conical dilator is used to expand the coil and allow the passage of the thickest possible locking stylet. A common guide for the pacemaker lead is passed at this time to assure that the lumen of the conductor is completely free as far as the distal portion of the lead, which is implanted in the myocardium, and also to provide an accurate measurement of the distance at which the locking stylet should be introduced. The internal diameter of the lumen of the conductor is then measured with a set of locking stylet measurers. The locking stylet with the greatest possible diameter is then introduced into the coil until the end of the lumen is reached and expanded, therefore, increasing the rigidity and the traction power of the lead. After this maneuver, a new traction may be applied to the lead to check whether its release from the myocardium is feasible without using countertraction.

If the electrode is not freed, the set of sheaths (the internal, which emits the laser light, and the external, of polypropylene) is applied to the lead, using it as a guide for their introduction. The applications of laser light are then started, each application lasting for 5 seconds, always accompanied by fluoroscopy. As the laser sheath progresses towards the heart, the external polypropylene sheath also advances, freeing the adherences of the lead to the venous and cardiac structures, until the most distal portion of the lead is reached by the laser sheath. At this point, the use of the laser light is interrupted, the laser sheath is used to provide circumferential support to the myocardium, and, applying traction to the lead, it is extracted from the myocardium. Then, the entire set (external sheath, laser sheath, and lead) is extracted, and hemostasis is performed at the site of introduction of the catheter by simple compression.

\section{Results}

Fifty-four catheters were extracted, resulting in a $96.4 \%$ rate of success. The extraction of 46 leads $(82.1 \%)$ was complete. In 8 other leads, only the metallic tip remained attached to the myocardium (14.3\%), and only 2 leads could not be extracted at all (3.6\%).

The duration of laser light application ranged from 20 to $540 \mathrm{~s}$ (mean $=123.0 \pm 104.5$ ), and 800 to 23,380 pulses $($ mean $=5,215.2 \pm 4,924.0)$ were applied, in a total of 4 to 119 cycles of application (24.4 \pm 24.2$)$.

The following mechanisms accounted for the release of the lead from the myocardium: 1) countertraction after complete progression of the laser sheath in 34 electrodes $(60.7 \%)$; 2) traction during progression of the sheath in 12 $(21.4 \%)$; 3 ) only traction after complete progression of the sheath due to the impossibility of countertraction (usually because of adhesion of the lead to the lumen of the sheath due to fibrosis) in $4(7.1 \%)$; 4 ) only traction due to failure in complete progression of the sheath in $2(3.6 \%)$, and other forms in 4 (7.1\%).

One patient had cardiac tamponade after extraction of the defibrillator lead, requiring open surgery on an emergency basis for correction of a laceration in the tip of the right ventricle.

The 2 unsuccessful cases were related to the presence of a large amount of calcium in the trajectory of the lead.

\section{Discussion}

In regard to extraction of leads, the state-of-the-art of artificial cardiac stimulation has revealed the advantages of the techniques that use internal countertraction when compared with other techniques.

The high rate of success obtained with the countertraction techniques with mechanical dilators has been reported in the literature. Smith et $\mathrm{al}^{3}$ have reported the following results obtained from 1988 to 1994 in the U.S. Lead Extraction Database: $86.8 \%$ of complete extraction; $7.5 \%$ of incomplete extraction; $5.7 \%$ of failure; $2.5 \%$ of severe complications; and $0.6 \%$ of mortality. Byrd et $\mathrm{al}^{4}$, analyzing data collected from January 1994 to April 1996 in the same database in a period when these techniques were considered reasonably stabilized, reported the results of extraction of 3,540 leads from 2,338 patients: $93 \%$ of complete extraction; $5 \%$ of partial extraction; $2 \%$ of failure in the procedure, and $1.4 \%$ of severe complications. Kantharia and Kutalek ${ }^{5}$ reported a 98\% rate of success in the extraction of pacemaker and defibrillator leads, with a $0.7 \%$ rate of severe complications, represented by 2 cases of cardiac tamponade.

Byrd et $\mathrm{al}^{4}$, analyzing the results of the U.S. Lead Extraction Database, concluded that the risk of failure in extraction or of incomplete extraction increases as follows: the longer the time the lead is implanted ( $\mathrm{p}<0.0001)$; the less experience the physician performing the procedure has $(p<0.0001)$; when the electrode is implanted in the ventricle $(\mathrm{p}<0.005)$; and the younger the patient is $(\mathrm{p}<0.0001)$. On the other hand, the risk of complications increases as follows: the higher the number of leads to be extracted $(\mathrm{p}<0.005)$; the less experience the physician performing the procedure has $(\mathrm{p}<0.005)$; and in patients of the female $\operatorname{sex}(\mathrm{p}<0.01)$.

According to Kantharia and Kutalek ${ }^{5}$, the major causes of failure of extraction are related to: a) the severity of the fibrous scar, which increases with the length of time that the lead is implanted; $b$ ) the experience of the physician performing the procedure; and c) the type of electrode to be extracted. According to these authors, active fixation atrial leads coated with silicone are completely extracted more frequently than passive fixation ventricular leads coated with polyurethane. Fibrosis can be very intense at 4 months after implantation of electrodes coated with silicone, and at 6 months for those coated with polyurethane ${ }^{5}$.

The use of laser light (replacing mechanical dilators) to allow the introduction of the sheaths for countertraction has resulted in a reduction in the duration of the procedure and has increased safety. 
In 1999, Wilkoff et al ${ }^{6}$ carried out a study in which 301 patients were randomized, using 2 techniques for countertraction, as follows: in 1 group, mechanical dilators were used, and, in the other group, the excimer laser was used. They reported that the use of the laser light allowed the following results: complete extraction of the leads in $94 \%$ of the cases; partial extraction in $2.5 \%$ of the cases; failure in $3.3 \%$ of the cases; and severe complications in $2.6 \%$ of the cases. They also reported a significant superiority $(\mathrm{p}=0.001)$ in the results obtained with the use of the laser light $(94 \%$ success for the laser-assisted procedure, and $64 \%$ success for the conventional techniques) in a significant number of patients, who, due to failure or difficulty in extracting the electrode by classical techniques, migrated to the group of patients randomized for laser-assisted extraction (crossover in 72 electrodes) ${ }^{6}$.

Kennergren ${ }^{7}$, analyzing the results obtained by the European Multicenter Study on excimer laser-assisted extraction with 179 catheters approached in 149 patients, has reported the following: complete extraction of $89.5 \%$ of the electrodes; partial extraction of $6 \%$ of the electrodes; failure of extraction of $4.5 \%$ of the electrodes; severe complications with $2.7 \%$ of the electrodes.

The results obtained in our study, when compared with those in the literature, show the reproducibility of the method.

The $96.4 \%$ rate of success and $2.8 \%$ rate of severe complications are in accordance with the rates found in the literature, even though the incidence of incomplete extraction (14.3\%) was higher in our study. Using the conclusions of Byrd et $\mathrm{al}^{4}$ and Kantharia and Kutalek ${ }^{5}$ and analyzing data shown in tables III and IV, we can observe that the population reported in our study has a higher risk of failure and of incomplete extraction than those populations studied by Wilkoff et $\mathrm{al}^{6}$ and Kennergren ${ }^{7}$.

Our patients are a mean of 11 and 14 years younger than those studied by Wilkoff et al ${ }^{6}$ and Kennergren ${ }^{7}$; the leads in our cases had clearly been in place longer (39\% and $32 \%$, respectively), and the proportion of atrial leads as compared with the ventricular ones was lower in our study

\begin{tabular}{|lccc|}
\hline \multicolumn{4}{|c|}{$\begin{array}{c}\text { Table III - Comparison of the results obtained with laser-assisted } \\
\text { extraction of catheters }\end{array}$} \\
\hline & $\begin{array}{c}\text { Wilkoff } \\
\text { et al., } 1999\end{array}$ & $\begin{array}{c}\text { Kennergren, } \\
1999\end{array}$ & InCor \\
\hline & $96.5 \%$ & $95.5 \%$ & $96.4 \%$ \\
Success & $94 \%$ & $89.5 \%$ & $82.1 \%$ \\
Complete extraction & $2.5 \%$ & $6 \%$ & $14.3 \%$ \\
Incomplete extraction & $3.3 \%$ & $4.5 \%$ & $3.6 \%$ \\
Failure & $2.6 \%$ & $2.7 \%$ & $2.8 \%$ \\
Severe complications & $2.6 \%$ & & \\
\hline
\end{tabular}

\begin{tabular}{|lccc|}
\hline \multicolumn{4}{|c|}{ Table IV - Comparison of the populations undergoing laser- } \\
assisted extraction of catheters \\
\hline & $\begin{array}{c}\text { Wilkoff } \\
\text { et al., 1999 }\end{array}$ & $\begin{array}{c}\text { Kennergren, } \\
1999\end{array}$ & Incor \\
\hline & 153 & 149 & 36 \\
\hline Number of patients & $65 \pm 18$ & 68.3 & $54.2 \pm 21.9$ \\
Mean age (years) & $65 \pm 42$ & 68.3 & $90 \pm 66$ \\
Permanence (months) & 1.59 & 1.2 & 1.55 \\
Leads/patient & $125 / 118$ & $104 / 57$ & $14 / 42$ \\
Atrial/ventricular & $33 \%$ & $35 \%$ & $39 \%$ \\
Female sex & & & \\
\hline
\end{tabular}

( 0.33 versus 1.06 and 1.83 , respectively). All these factors suggest a higher risk of complications or of partial extraction. The other 2 criteria observed, number of leads per patient and proportion of female patients, have similar figures for the 3 populations analyzed.

In conclusion, the use of the excimer laser has allowed extraction of catheters with a high success rate and a low level of complication. These complications, however, tend to be severe, requiring immediate action to save the patient's life, including the possibility of open thoracic surgery.

\section{Acknowledgements}

We thank Anísio Pedrosa, João Luiz Piccione, Neide Romão, Sérgio Siqueira, Silvana D’Ório Nishioka, and WagnerT. Tamaki.

\section{References}

1. Byrd CL, Shwartz SJ, Hedin N. Lead extraction: indications and techniques. Cardiol Clin 1992; 10: 735-48.

2. Klug D, Lacroix D, Savoye C, et al. Systemic infection related to endocarditis pacemaer leads. Clinical presentation and management. Circulation 1997; 95: 2098-107.

3. Smith HJ, Fearnot NE, Byrd CL, Wilkoff BL, Love CJ, Sellers TD. Five-years experience with intravascular lead extraction. U.S. Lead Extraction Database. Pacing Clin Electrophysiol 1994; 17: 2016-20.

4. Byrd CL, Wilkoff BL, Love CJ, et al. Intravascular extraction of problematic or infected permanent pacemaker leads: 1994-1996. U.S. Extraction Database, MED Institute. Pacing Clin Electrophysiol. 1999; 22: 1348-57.

5. Kantharia BK, Kutalek SP. Extraction of pacemaker and implantable cardioverter defibrillator leads. Curr Opin Cardiol. 1999; 14: 44-51.

6. Wilkoff BL, Byrd CL, Love CJ, et al. Pacemaker lead extraction with the laser sheath: results of the pacing lead extraction with the excimer sheath (PLEXES) trial. J Am Coll Cardiol. 1999; 33: 1671-6.

7. Kennergren C. Excimer laser assisted extraction of permanent pacemaker and ICD leads: present experiences of a European multi-centre study. Eur J Cardiothorac Surg 1999; 15: 856-60. 